\section{Janko Gravner and Kyle Johnson}

Mathematics Department

University of California

Davis, California 95616, USA

\{gravner, kyljohnson\}@math.ucdavis.edu

\title{
Coupled Map Lattices as Musical Instruments
}

\begin{abstract}
We use one-dimensional coupled map lattices (CMLs) to generate sounds that reflect their spatial organization and temporal evolution from a random initial configuration corresponding to uncorrelated noise. In many instances, the process approaches an equilibrium, which generates a sustained tone. The pitch of this tone is proportional to the lattice size, so the CML behaves like an instrument that could be tuned. Among exceptional cases, we provide an example with a metastable strange attractor, which produces an evolving sound reminiscent of drone music.
\end{abstract}

A common mathematical model of a complex feedback system is iteration of a nonlinear map of one or more variables. The resulting unpredictability and diversity of behavior naturally invite possible connections to music, where such models have indeed found uses, primarily to generate note patterns for use in compositions (e.g., Battey 2004). In this article we instead adopt this approach to produce a single sound whose characteristics reflect the evolution induced by a chosen recursive rule.

We focus on coupled map lattices, a class of iterative systems built from a large number of identical functions of a single variable linearly coupled to nearest neighbors in a network. As has been known since their inception, these are particularly convenient models to study self-organization: From a disordered initialization, many instances exhibit rapid convergence to a steady state with recognizable spatial patterns (Kaneko 1993). Our main goal, then, is to represent these patterns as sounds.

Note that, in this article, we use the term "space" to refer to the abstract space in which the evolving array of variables is arranged, not to the physical space of a listening environment or of a musical instrument.

\section{Spatial Organization and Sound Synthesis}

Suppose we have a system that evolves in time, and we want to use it to generate a sound stream. Such a system possibly has a high-dimensional set of variables, together with one-dimensional time, and

Computer Music Journal, 42:2, pp. 22-34, Summer 2018 doi:10.1162/COMJ_a_00458

(c) 2018 Massachusetts Institute of Technology. for sound generation this multidimensional array needs to be reduced to a one-dimensional sequence of numbers. One way to achieve this is to simply produce one signal per time step, as a single function of all variables of the system (Stefanakis, Abel, and Bergner 2015). There might be a canonical selection of such a function in special cases, but in general the choice is necessarily arbitrary. Moreover, although this method does reveal temporal evolution (at least if the sampling variable is carefully chosen), any spatial organization is not captured. To remedy this, we seek to include every variable in our output sequence.

To investigate spatial organization, we must have space, and a particularly useful role is played by the space $\mathbb{Z}^{d}$ of integer vectors with $d$ coordinates, the $d$-dimensional integer lattice. An evolving configuration attaches a variable, in our case a number, to each point in the lattice, and uses a rule to modify the variables over time. We will assume that $d=2$, as the same argument applies equally in higher dimensions. Then there is no natural way to "cut the configuration into onedimensional ribbons" to generate a sound stream that would incorporate every variable, not even at a single time. In short, the problem is that the time (for the sound sequence) is a priori linearly ordered, whereas the two-dimensional lattice is not. Nevertheless, there have been some attempts based on two-dimensional cellular automata such as the Game of Life and voter models (Burraston and Edmonds 2005; Miranda 2007; Serquera and Miranda 2011, 2014).

We conclude that the most promising approaches are processes on $\mathbb{Z}$, i.e., those whose configuration is a finite array of numbers arranged on an integer interval, say $[0, n-1]$. The number $n$ is the lattice size. Referring to the space, we call such processes one-dimensional, although the dimension of the 
configuration space equals $n$ and could be very large, typically in our examples between 50 and 400 .

The configuration of our process at a time $t$ therefore consists of real numbers $x_{t}(0), \ldots, x_{t}(n-$ 1). This process will evolve in discrete time $t=$ $0,1,2, \ldots$ according to a rule that will be specified shortly, but first we reveal how we obtain the sequence used to generate sound. Namely, we form the unique sequence of variables that preserves the order of the time, and then the order of the one-dimensional lattice:

$$
\begin{aligned}
& x_{0}(0), \ldots, x_{0}(n-1), x_{1}(0), \ldots, x_{1}(n-1), \ldots, x_{t}(0), \ldots, \\
& x_{t}(n-1), \ldots
\end{aligned}
$$

\section{Coupled Map Lattices}

Particularly appealing update rules to study selforganization are those with very simple local interaction. We now introduce one such class of rules, which posit that the variable at a location $i$ is first updated using a function, possibly one that is nonlinear, of only its current state; the value at the next time step is computed by a weighted average of its value and those of its nearest neighbors in the one-dimensional lattice. Thus the second step is linear and any perturbation spreads at a speed of at most one, which is often called the "light speed." That is, $x_{t}$ is given recursively, for each time $t=0,1,2, \ldots$, by the rule

$$
x_{t+1}(i)=(1-\epsilon) f\left(x_{t}(i)\right)+\frac{\epsilon}{2}\left[f\left(x_{t}(i+1)\right)+f\left(x_{t}(i-1)\right)\right] .
$$

Here, $\epsilon \in[0,1]$ is the coupling strength that determines the influence of spatial neighbors, and $f$ is a given function. We assume periodic boundary, that is, for $i \in[0, n-1]$, its neighbors $i-1$ and $i+1$ are computed modulo $n$. Such a rule is called a onedimensional coupled map lattice (CML). We will restrict the variable values $x_{t}(i)$ to a suitable interval, either $[-1,1]$ or $[0,1]$. In the latter case, whenever the updated variable is outside the interval we replace it with its fractional part; equivalently, we could assume that individual variables are points on a (continuous) circle.
Typically, our initial configuration is random. To be precise, $x_{0}(i), i=0, \ldots, n-1$ are chosen independently and uniformly from the set of possible values. This maximally disordered initialization is arguably the best test of the rule's self-organizing capabilities. Interesting sounds can be generated with special initial configurations, however, as we will see later in sections on "Sine Wave Initialization" and "Zero Initialization."

Coupled map lattices were utilized by Kunihiko Kaneko in 1983 to model spatiotemporal chaos with discrete-time, discrete-space, and continuous-state dynamics (for a survey, see Kaneko 1993). We will only consider three examples of the map $f$ : the linear map, the logistic map used by Kaneko, and the circle map. For the latter two choices, CMLs are, in a sense, coupled nonlinear oscillators. The linear map facilitates the exposition through its simplicity, and demonstrates an interesting connection between CMLs and the Karplus-Strong algorithm. The logistic map is considered because of the role it has played in the existing literature on CMLs, and because it provides good examples of periodic, intermittent, and chaotic behavior. The most interesting sounds, however, are generated by the circle map, which exhibits a number of interesting evolutions. Examples we provide include chaotic, periodic, and almost periodic behavior; synchronization; interacting particles; and metastable strange attractors. Often, the mixture of noise and order in the CML is reminiscent of chimera states in neural network dynamics (Hizanidis et al. 2016); see, for example, the CML given later in the section on "Evolution of CML with the Circle Map: Metastable Strange Attractor."

There are several natural ways in which Equation 2 can be generalized. For example, one may change the neighborhood of $i$ from nearest neighbor $i \pm 1$ to a larger range $r: i \pm 1, \ldots, i \pm r$. This does not seem to make a qualitative difference. Another possibility is to forgo translation invariance and to assign either a different $\epsilon$ or a different $f$ to different lattice points $i$. A random assignment of this kind is an analogue of spin-glasses (Stein and Newman 2013) and is an intriguing direction that is largely beyond the scope of this article, although we do provide one example (see Equation 3 later in this 
article). Finally, generalizations to other lattices are mathematically interesting, but, as discussed, it is unclear whether this could be done without losing the natural correspondence to digital audio signals.

\section{Previous Work}

Of all existing sound-synthesis techniques, our approach most closely resembles the linear automata synthesis (LASy) of Jacques Chareyron (1990). There are two main differences between LASy and CML synthesis. The first is that the former uses cellular automata with a large set of discrete variable values, whereas the latter uses coupled map lattices, which have continuous variables. In this sense, our approach is a natural extension of LASy. The second difference is that the LASy method focuses on linear cellular automata, mentioning other possibilities only in passing, whereas we view linear rules as a starting point into more general CMLs. Notice again that both of these schemes use one-dimensional lattices; higher-dimensional cases require mapping the output of the system in a more creative way. For example, histogram mapping synthesis (Serquera and Miranda 2011,2014) uses densities of variables in evolving cellular automata dynamics to control the parameters of a synthesizer. Another approach, Chaosynth, uses a cellular automaton inspired by neural reverberatory circuits to control the pitch and duration of sinusoidal grains in a granular synthesizer (Miranda 1995).

A recent article by Stefanakis, Abel, and Bergner (2015) uses ordinary differential equations (ODEs) for digital synthesis. This method bears some similarity to ours in that it uses dynamical systems. It generates only one signal per time step, as a function of all variables, to generate a time series that produces the sound, however. By contrast, each of our variables generates a signal in the lattice order, so the resulting sound reflects spatial organization as well as temporal evolution. Furthermore, discrete space and time have the practical advantage that computations are simple and very fast. By contrast, coupled ODEs (which evolve in discrete space and continuous time) or partial differential equations (in which both space and time are continuous) would require potentially sophisticated solvers (Dzjaparidze 2015).

There is also a theoretical advantage to CMLs: No existence and uniqueness results are needed.

\section{Key Features of CML Synthesis}

In our experiments, most CML dynamics rapidly converge to a stable equilibrium, which results in a steady tone. In almost all cases, the pitch of the tone is inversely proportional to the lattice size. The proportionality constant depends on the sampling rate and the temporal behavior of the CML. Although we do not have a formal definition, we refer to these cases as regular. Two exceptions are discussed in some detail later in this article: a CML with a logistic map (Equation 4) with a highly chaotic behavior (producing white noise), and a CML determined by the parameters in Equation 11 with a highly organized behavior (resulting in a high frequency tone). Both of these require very specific parameter settings.

In the regular cases, the timbre of a CML's tone is determined by the chosen parameters and the initial configuration. The random initialization results in slight variations between different runs of the dynamics, akin to physical instruments, which also produce subtly different tones even when the same pitch is played with the same loudness. The effect of the parameters on the timbre of a CML is dependent on the definition of the map, but some general observations can be made. The degree to which the map $f$ itself is chaotic has the expected effect. Namely, the nonlinearity parameter $(a$ in the logistic map, defined later in Equation 4, and $K$ in the circle map, defined in Equation 5) governs how simple the sound is: Large nonlinearity results in chaotic noise, and other values generate tones with varied degrees of complexity. The coupling strength $\epsilon$ has an unpredictable effect on evolution, and often needs fine tuning.

\section{Outline and Highlights}

Our main purpose is to use CMLs as instruments in the following fashion: Generate a random initial 
configuration, choose the dynamic parameters, and observe the resulting sound. Different choices produce starkly different sounds, from pitched tones, to noise, to something in between. In the following sections, we concentrate on examples, both to illustrate the regular dynamics and to provide interesting unusual cases. First, however, we give an overview of our approach in the following section. We follow with a brief exposition on the basic case of the linear map, and then provide three examples with the logistic map that demonstrate how CMLs generate sound that range between order and noise. Perhaps the most interesting are the CMLs that use the circle map $f$. We provide four examples: The first demonstrates that CMLs are capable of producing rich and varied textures thanks to slow convergence to the steady state, the second is an example of a nonunique equilibrium with unusual lack of dependence on the lattice size, the third produces slowly changing timbre, and the last CML generates a frequency that is lower than expected. Finally, we give a few examples with special initializations. We demonstrate the use of CMLs as musical instruments by tuning them to play a few melodies, some well-known and some computer-generated. Three example melodies are described in the Appendix, including a description of the parameters used.

\section{Interpreting a CML Evolution as a Sound}

As explained in the section "Spatial Organization and Sound Synthesis," we interpret each lattice variable in a CML evolution as a digital audio signal, and order these signals as in Equation 1. We have chosen a sample rate of $44.1 \mathrm{kHz}$ (the compact disc standard) in all our examples. After a short burst of white noise (due to the initial randomness), the resulting audio stream is a sound with evolving timbre. Because the dynamics tend to rapidly evolve into a stable or metastable steady state, the transient period is almost imperceptible and the result is close to a single sound. The nature of the sound depends upon the behavior of the CML. If the equilibrium is spatially simple, dominated by temporarily periodic regions, our synthesizer produces a pitched tone.
Spatial simplicity alone does not suffice for a pitched tone, however. As we will see in the section on "Zero Initialization," there is an example with nearly constant equilibrium whose values change chaotically and hence result in noise, even with constant initialization. On the other hand, it is easy to generate simple temporal behavior (namely, by choosing $f$ to be the identity map with $\epsilon=0$ ), which leaves the spatial chaos invariant and, hence, also produces noise.

We reiterate that two sounds, generated with the same map, parameters, and lattice size, but different random initial samples, will both sound similar, as they are generated by the same process, but they are subtly distinct from one another. This balance of similarity and variation is analogous to the way sounds produced by a physical instrument will have a recognizable timbre, but will reveal subtle differences when sounding the same note. Another parallel is that the dominant frequency, and therefore the tuning procedure, cannot be determined a priori for all parameter choices, but only after running the particular dynamics-by "playing the instrument," as it were. We elaborate on this point next.

With the exception of a few special cases, the pitch of the tone produced by a CML is inversely proportional to the lattice size $n$. Given a CML with a lattice of size $n$ and a sample rate of $s$ samples per second, the fundamental frequency of the tone produced by the CML synthesizer will be $s /(n T)$ $\mathrm{Hz}$ for some $T \in\{1,2,3, \ldots\}$. If a CML has a steady state that is periodic (or nearly periodic) with a sufficiently small temporal period, then $T$ will be determined by the temporal period of the CML. This is because the sequence of samples generated by the consecutive lattice points will be periodic (or nearly so) with period $n T$. and so they will produce a tone of frequency $s /(n T)$. If the temporal period is too large, $s /(n T)$ will be below the range of human hearing and we will perceive the strongest upper partial of this frequency as the fundamental. In this case, the dominating frequency tends to be $s / n$.

The only exceptions to the linear dependence on $n$ that we are aware of involve rules that produce a very close approximation to white noise and rare spatially periodic examples. Even chaotic 
sounds that do not produce a tone having a clear, definite musical pitch do have an audible change of pitch as a result of a change in the lattice size. Additionally, the discontinuity between temporal updates provides a periodic signal with frequency inversely proportional to the lattice size. These two contributions are fundamentally different. For example, if $n$ is large, the second effect amounts to a sequence of "pops." To reiterate, the inverse of $n$ will be present in the frequency domain, except when the lattice configuration is spatially periodic and temporarily constant. The latter level of regularity is very difficult to achieve with a CML starting from a random initialization. We do, however, provide an example in which this happens at least with a positive probability (this will be discussed in greater detail in the section "Evolution of CML with the Circle Map: Annihilating Diffusions"). Trivially, we can also achieve this by the identity map $f, \epsilon=0$, and with a periodic initial configuration.

The diffusion caused by the local averaging acts, in the frequency domain, as a low-pass filter on the signal after the possibly chaotic map $f$ has been applied to each lattice point. This filtering operation mitigates the extent to which higher partial tones are present in the sound produced by the CML and thus helps to reinforce the fundamental frequency proportional to the inverse of $n$. The strong and predictable relationship between the lattice size and the frequency of the sound enables CMLs to be used as a natural method of digital synthesis.

For example, a tone generated by a CML with temporal period 2 and lattice size 50 at $44.1 \mathrm{kHz}$ will have a fundamental frequency of $441 \mathrm{~Hz}$, which is about 4 cents sharper than the middle $\mathrm{A}$ tuned at $440 \mathrm{~Hz}$. Notice that with the standard $44.1-\mathrm{kHz}$ sample rate, we cannot get closer to the frequency $440 \mathrm{~Hz}$. The relationship between $n$ and frequency allows us to easily control the pitch generated by a CML, but it also causes the precision of our tuning to be limited by the sample rate.

\section{A Simple Example: The Linear Case}

Consider a linear map $f:[-1,1] \rightarrow[-1,1]$, defined by $f(x)=a x$ for all $x \in[-1,1]$. Here, the coefficient $a$ is in $[-1,1]$ (values of $a$ outside of this range can be used, but then we have to use a fractional part operation to map back into the interval $[-1,1])$. If we start from a random initialization and take $\epsilon \in(0,1]$, this gives us a CML which is, effectively, an implementation of the Karplus-Strong algorithm for plucked-string synthesis (Karplus and Strong 1983). Indeed, the transition rule of our CML takes a weighted average of adjacent samples in the sound stream generated by Equation 1, and thus acts as a low-pass filter on the signal.

This linear case is particularly attractive for the following reason: When it starts from randomness, it emulates a plucked string of length proportional to the length of lattice. In fact, this case has appeared in the literature in the guise of a linear cellular automaton with a large number of variable values (Chareyron 1990). A few examples generated using $a=0.99$ and $\epsilon=0.5$ are illustrated visually in Figure 1 and can be heard as Sound Examples 1-3. [Editor's note: All sound examples can be heard on the CMI Web site at www.mitpressjournals.org/doi/suppl/10.1162 /COMJ_a_00458.]

In Figure 1a (and in later figures), we exhibit space-time pictures of the corresponding CML evolutions. As is standard in the CML literature, the entire spatial configurations $x_{t}(0), \ldots, x_{t}(n-1)$ are depicted horizontally, with the configuration at time $t+1$ below the one at time $t$. Thus, the initial state at time $t=0$ is at the top, and time runs downwards. The variable at each lattice point is linearly mapped to a shade of gray, so that white corresponds to the right endpoint of the state space and black to the left endpoint. For example, in Figure 1a the variable is in $[-1,1]$, so the lighter and darker shades depict values close to 1 and -1 , respectively.

Figure $1 \mathrm{~b}$ displays a spectrogram of the sound generated by the same CML, with lighter shades of gray corresponding to larger amplitudes, using the same time period as the space-time pictures. In this (and the following figures), time runs horizontally from left to right, as is standard for spectrograms.

We also provide a preliminary example of the disordered version of the CML rule given by Equation 2, namely, 
Figure 1. The evolution of the linear coupled map lattice (CML) with lattice size $\mathrm{n}=100, \mathrm{a}=0.99$, and $\epsilon=0.5$, from a random initial configuration, in the time domain (a) and in the frequency domain (b). The time-domain

representation used here places time on the vertical axis, oriented downwards, and space on the

horizontal axis, as is typical in the $C M L$

literature.

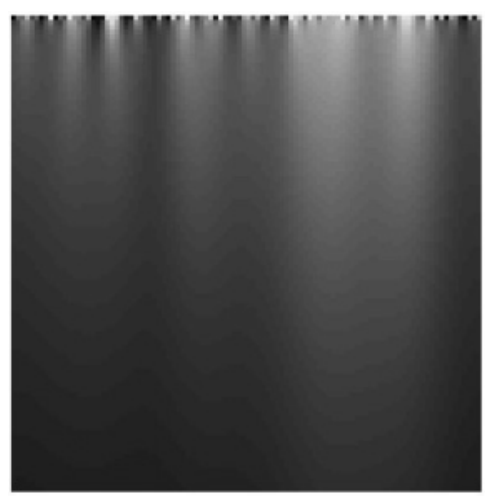

(a)

$x_{t+1}(i)=\left(1-\epsilon_{i}\right) f\left(x_{t}(i)\right)+\frac{\epsilon_{i}}{2}\left[f\left(x_{t}(i+1)\right)+f\left(x_{t}(i-1)\right)\right]$,

where $\epsilon_{i}$ are random, in our case chosen independently and uniformly on $[0,1]$. This produces a sound with more pronounced upper partials that correspond to enhanced horizontal lines in the spectrogram in Figure 2; refer also to Sound Examples 4-6.

\section{The Logistic Map}

We use the following version of the logistic map:

$$
f(x)=1-a x^{2} .
$$

We will assume that the nonlinearity parameter $a$ is in the interval $[1,2]$, so that $f$ maps the interval $[-1,1]$ into itself. The choice of Equation 4 was made by Kaneko in his pioneering work on CMLs (Kaneko 1993). Other versions of the logistic map are equally natural and produce similar results (e.g., $f(x)=a x(1-x)$ ).

Values of $a$ near 1 result in a sound that rapidly converges to a steady tone. As $a$ increases, the CML behaves more chaotically, resulting in sounds that become closer to noise than to a pitched tone. See Figure 3 for time domain and spectrogram plots of logistic map CMLs with a few different parameter values. (Refer to Sound Examples 7-15.)

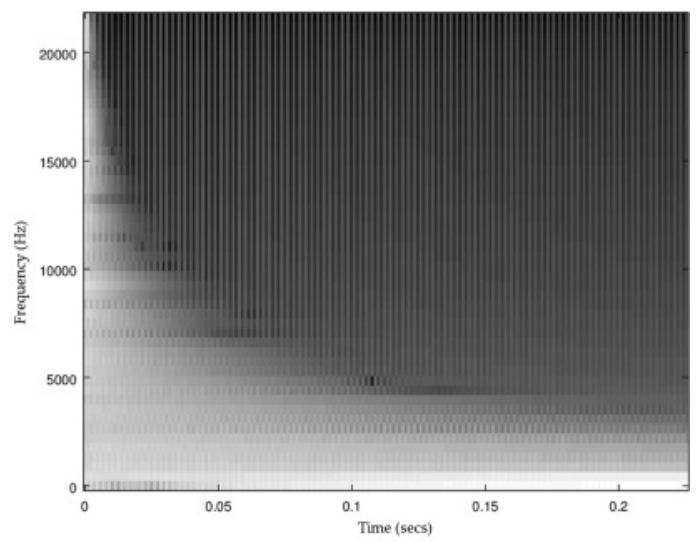

(b)

We note that when $a=2$ and $\epsilon=0$, each lattice point behaves chaotically and independently of others, resulting in a close approximation to white noise. This is a simple instance in which the lattice size has no effect on the sound produced by the CML.

\section{The Circle Map}

In this case, the variables have values in the interval $[0,1]$ and

$$
f(x)=x+\omega-\frac{K}{2 \pi} \sin (2 \pi x) \bmod 1 .
$$

Here, $\omega$ represents a phase shift and $K$ the strength of nonlinearity. Taking the fractional part $\bmod 1$ is needed because without it $f$ does not necessarily map $[0,1]$ into $[0,1]$. In the following discussion, we examine the behavior in the case of four different parameter settings with random initial configurations and explore special initializations.

The Kuramoto model is an ODE that has been widely studied as a prototype for synchronization, whereby the evolution leads to nearly equal variable values across space in spite of the possibly chaotic temporal evolution (for a comprehensive survey, see Acebrón et al. (2005); for examples, see our discussions of the metastable strange attractor and near-periodicity later in this article). With the circle map $f$ as in Equation 5, the CML could be 
Figure 2. The evolution of the disordered CML given by Equation 3, with lattice size $\mathrm{n}=100, \mathrm{a}=0.99$, and $\epsilon_{i}$ chosen independently and uniformly on $[0,1]$, from a random initial configuration, in the time domain (a) and in the frequency domain (b).

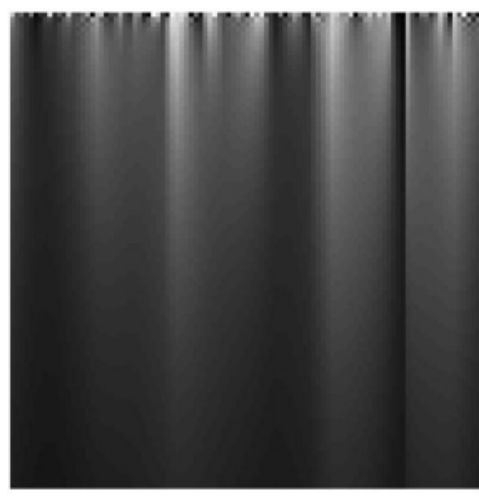

(a)

interpreted as a version of the Kuramoto model with discrete time and local coupling. Thus it is no surprise that we observe synchronization in several of our examples (see our remarks after Equations 6 and 13), although conditions on the parameter values that result in this scenario are rather unclear.

Evolution of CML with the Circle Map: Metastable Strange Attractor

First, we consider the circle map with parameters

$$
\omega=5 / 9, K=1, \epsilon=0.3 \text {. }
$$

Owing to the complexity and longevity of its evolution to the steady state, we need to develop some statistical tools to understand this CML. Figure 4 depicts the behavior of three statistics. The simplest is the unscaled variance

$$
\delta_{t}=\sum_{i=0}^{n-1} x_{t}(i)^{2}-\frac{1}{n}\left(\sum_{i=0}^{n-1} x_{t}(i)\right)^{2},
$$

which simply measures how far $x_{t}$ is from a constant. A more involved tool is the cross-correlation (Stoica and Moses 2005), defined for $t=1,2, \ldots$ by

$$
\operatorname{cross}_{-} \operatorname{corr} t(k)=\sum_{i=0}^{n-1} x_{t-1}(i-k) x_{t}(i) \text {. }
$$

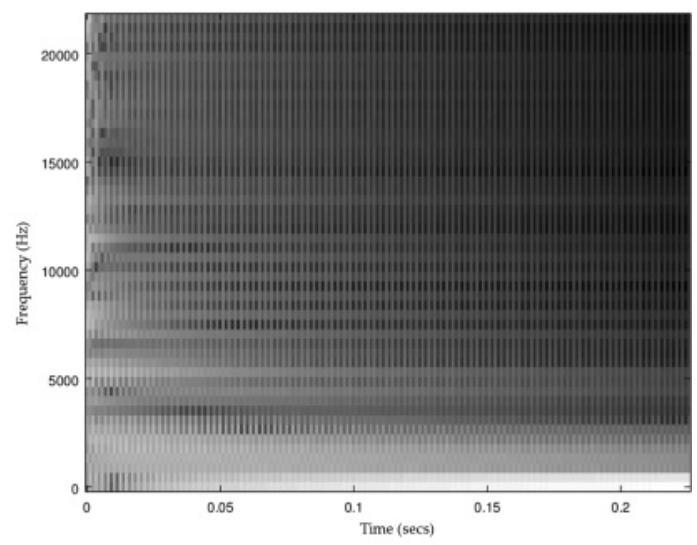

(b)

Note that, when negative, $i-k$ is interpreted as $i-$ $k+n$ because of the periodic boundary conditions. This quantity compares a configuration with its spatial shift by $k$, and leads to two other statistics:

$$
\begin{aligned}
\rho_{t} & =\max _{k} \text { cross_corr } r_{t}(k), \\
\tau_{t} & =\min \{k: \text { cross_corr } \\
t & \left.(k)=\rho_{t}\right\} .
\end{aligned}
$$

The quantity $\rho_{t}$ measures the extent to which $x_{t}$ is a translation of $x_{t-1}$, and $\tau_{t}$ is the optimal translation, that is, one with largest cross-correlation. See Figure 4 for the resulting evolution for three lattices sizes, up to time $10^{7}$. By this time, the dynamics with $n=70$ reached a stable synchronized configuration, with $\delta_{t}$ very close to 0 . Therefore, at any fixed time, all the variables are nearly equal. Their approximately common value, however, changes chaotically over time, owing to the nonlinearity of $f$. The resulting sound evolves into chaotic taps because of the discontinuities between iterates. A tone with frequency proportional to $1 / n$ is also heard (see the discussion in the section on "Interpreting a CML Evolution as a Sound"). We conjecture that this is the fate of the CML with parameters given by Equation 6 for every lattice size. The waiting time for $n \geq 100$ is so large, however, that synchronicity is in practice never reached. Instead, for $n$ between about 100 and 300 we see the dynamics largely oscillating between two metastable polarity configurations, characterized by waves traveling in two opposite directions, with brief periods of polarity reversal 
Figure 3. Three examples of the CML with the logistic map (Equation 4), each with 100 iterations and lattice size $\mathrm{n}=100$. Time-domain plots are given on the left, with spectrograms of the signal generated by the same CML on the right. The parameter values used are: $\mathrm{a}=1.1, \epsilon=0.3(a) ; \mathrm{a}=$ 1.75, $\epsilon=0.3(b)$; and $\mathrm{a}=2$, $\epsilon=0.5$ (c). Sound
Examples 7-15 are generated by these three pairs of parameters (see main text for the URL to the audio files).
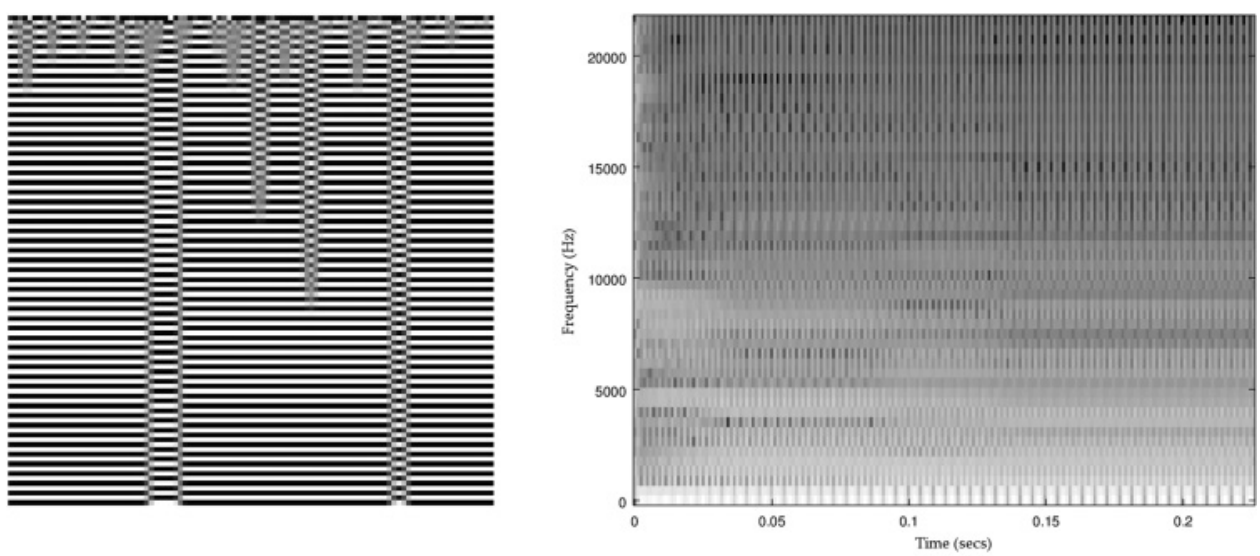

(a)
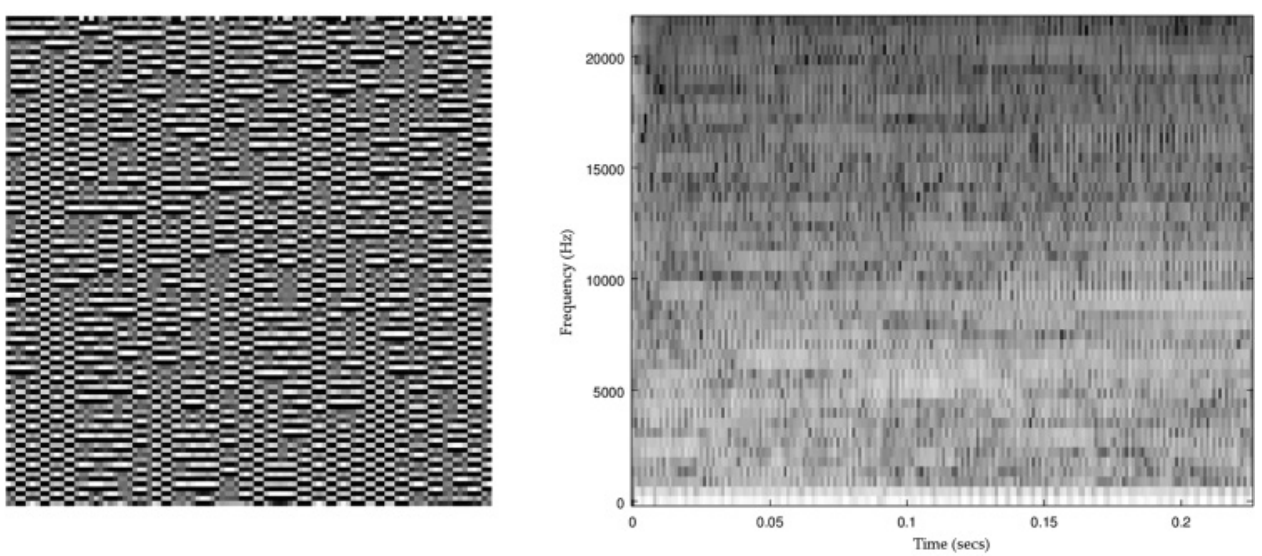

(b)
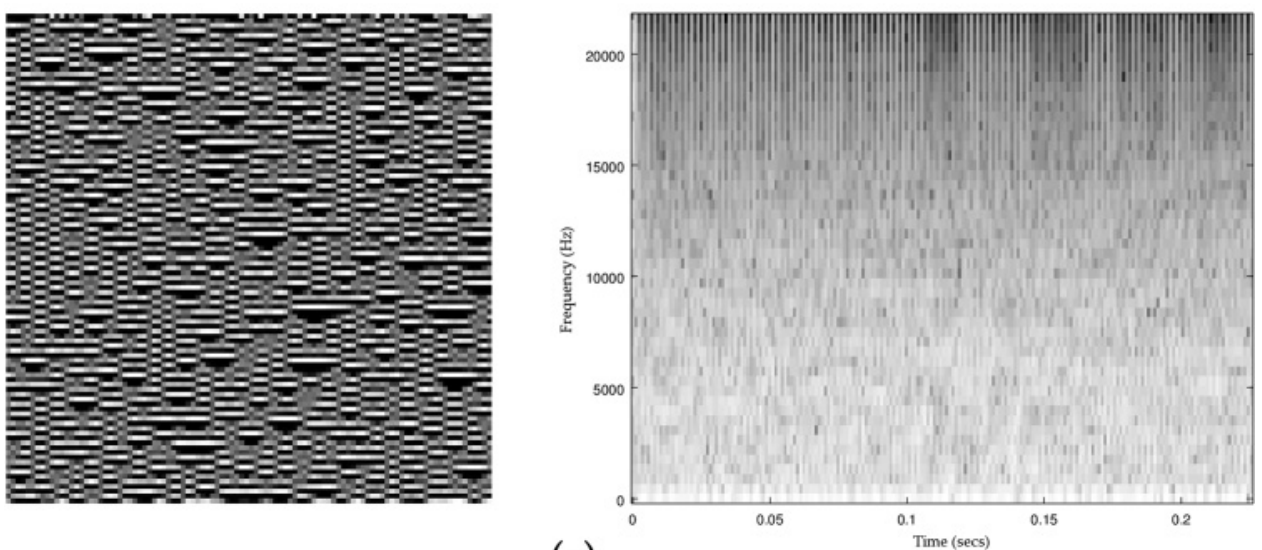

(c) 
Figure 4. Evolution of the $C M L$ with the circle map (Equation 5) and parameters given by Equation 6, with $\mathrm{n}=70$ (a), $\mathrm{n}=200(\mathrm{~b})$, and $\mathrm{n}=$ 400 (c). In each frame, the bottom (medium gray) curve depicts $\delta_{\mathrm{t}}$, the middle (dark gray) $\rho_{\mathrm{t}}$ and the top (light gray) $\tau_{\mathrm{t}}$. See text for complete explanation.

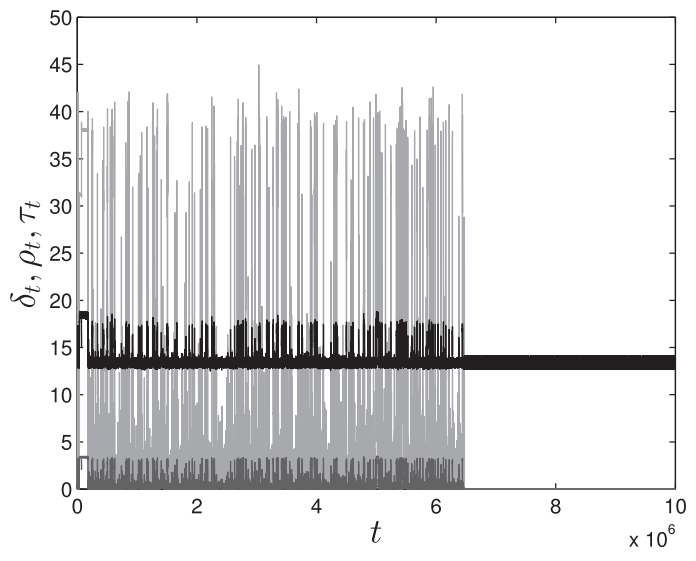

(a)

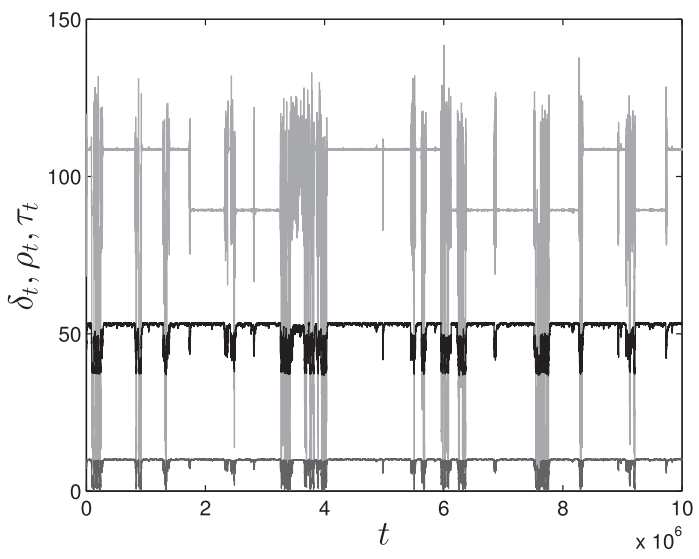

(b)

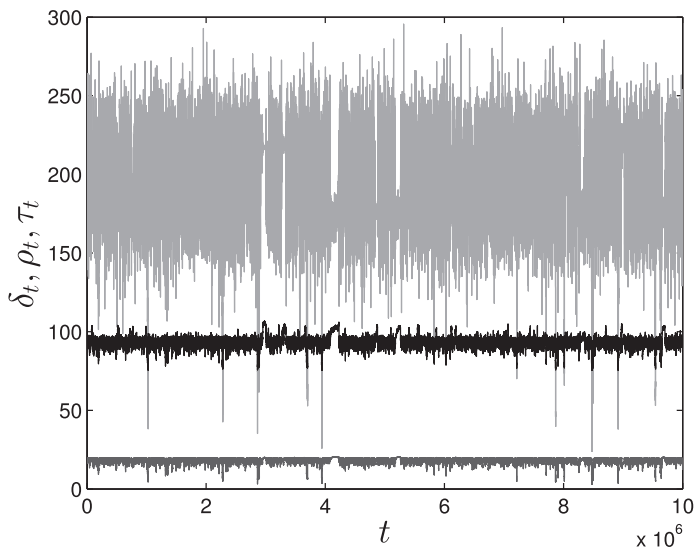

(c)

between them. This is most clearly seen in the middle frame of Figure 4, where polarity periods are distinguished by flat lines in the translation statistic $\tau_{t}$. The evolution is thus dominated by a kind of strange attractor that creates an evolving sound reminiscent of drone music (particularly of popular German electronic music of the 1970s, nicely exemplified on the Tangerine Dream album Zeit) punctuated by rhythmic crackling noises during reversals. For larger $n$, the temporal evolution appears chaotic in Figure 4, as the polarity is only rarely reached; however, the spatial organization is similar to that of the polarity configurations and thus the droning sound persists with no, or only rare, reversals.
Although these properties make these parameter settings inappropriate for use in a melodic or harmonic context, they result in a rich evolving timbre that is well-suited for avant-garde compositions. (Refer to Sound Examples 16-18.)

\section{Evolution of CML with the Circle Map: Annihilating Diffusions}

We now consider the case with parameters

$$
\omega=0.5, K=1, \epsilon=1 .
$$


Figure 5. The first 1,000

time steps of the evolution

of the CML with the circle

map (Equation 5) and

parameters given by

Equation 11, with $\mathrm{n}=400$.
Figure 6. First 250 time steps (a), followed by 500 time steps starting later around time step 40,000 (b), of the evolution of the
$C M L$ with the circle map (Equation 5) and parameters given by Equation 12, with $\mathrm{n}=400$.

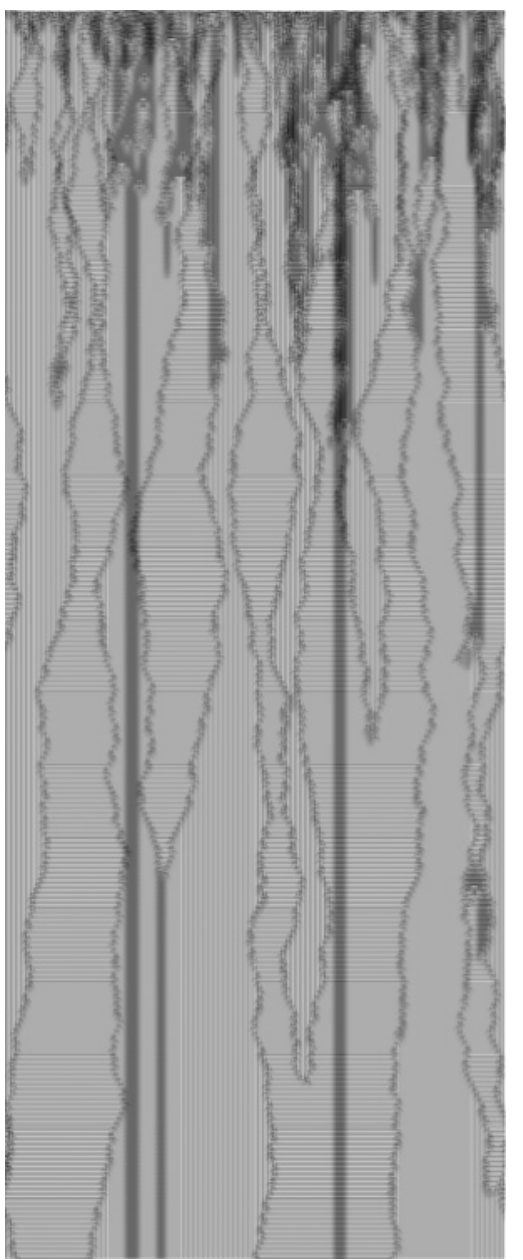

These generate two competing equilibria, one with horizontal stripes (with temporal period 2 and spatial period 1) and one with vertical stripes (with temporal period 1 and spatial period 2), with diffusing boundaries between the two equilibria. See Figure 5 for an illustration. There are also stationary defects that generate pronounced vertical lines and that interact with the boundaries in an unpredictable fashion. The invariant state is therefore not unique: One or the other equilibrium is reached with equal probability. When the lattice size is small, it is very likely that the stationary defects disappear and so, with probability close to $1 / 2$, the generated sound is a square wave with frequency equal to half the sample rate; this sound is very high-pitched and (a)

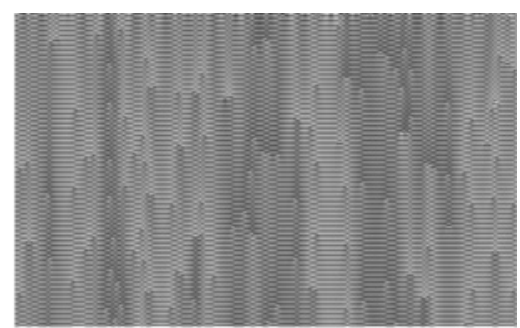

(b)

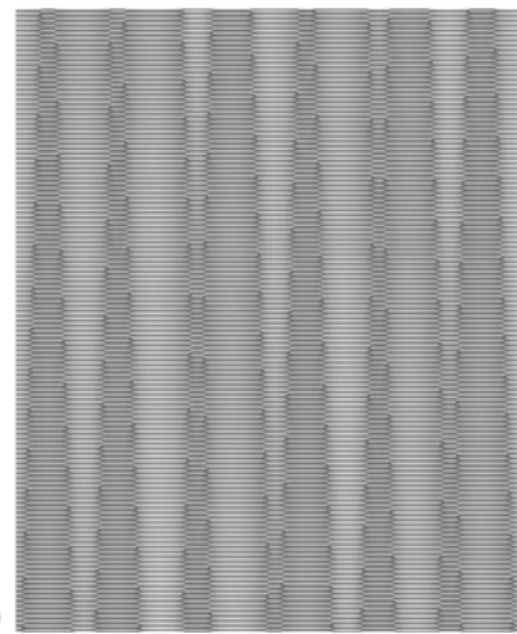

independent of the lattice size. (Refer to Sound Examples 19 and 20.)

\section{Evolution of CML with the Circle Map: Repelling Particles}

Next is the case with parameters

$$
\omega=0.5, K=0.1, \epsilon=0.6 \text {. }
$$

After a considerable time, the evolution selforganizes into a system of repelling particles on a slowly evolving background. Figure 6 provides an illustration. As a result, the tone produced by the CML with these parameters will have steadily changing timbre. Because the repelling particles travel slowly, a high degree of structural similarity is preserved between consecutive lattice variables, and so the tone evolves smoothly. After an initial period during which the particles are created and destroyed, their number stabilizes, which ensures an upper bound on the difference between two 
Figure 7. The first 250 time steps (a), followed by 500 time steps starting later around time step 40,000 (b), of the evolution of the
CML with the circle map (Equation 5) and parameters given by Equation 13, with $\mathrm{n}=400$. (a)

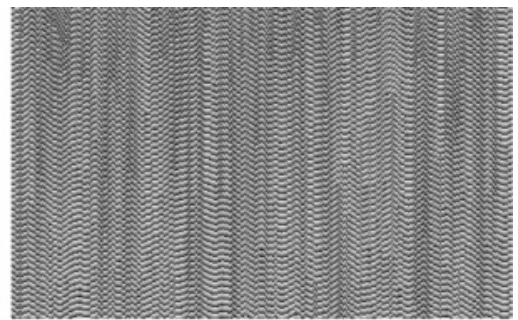

(b)

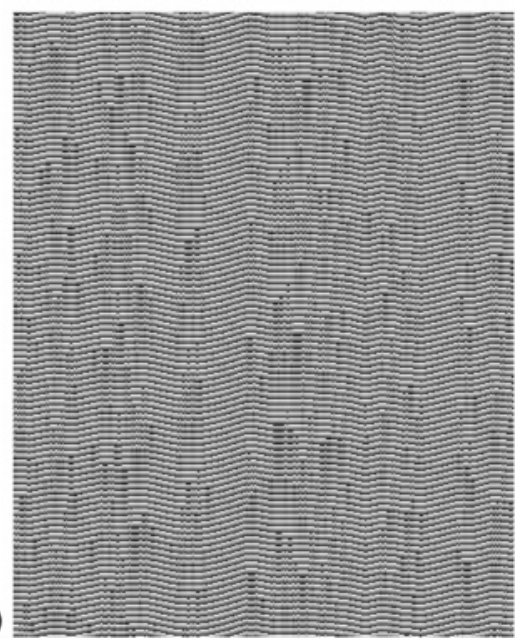

timbres at different times. This can be heard in Sound Examples 21 and 22.

\section{Evolution of CML with the Circle Map: Near-Periodicity}

Finally, we consider the case with parameters

$$
\omega=0.2, K=0.2, \epsilon=0.12 .
$$

This selection leads, after a few thousand iterations, to a stable, nearly periodic equilibrium with a spatial period of about 80; see Figure 7 . We note that $\epsilon$ requires some finetuning here: A significantly larger value for $\epsilon$ leads to a chaotic state, whereas a significantly smaller $\epsilon$ leads to synchronization. Unlike in the CML determined by Equation 6, however, the approach to synchronicity is gradual. The sound produced by these parameters has a timbre that varies somewhat more than the repelling-particle case given by Equation 12, but considerably less than the metastable strange-attractor case given by Equation 6. In addition to being nearly periodic spatially, these dynamics are also close to being temporally periodic, with a temporal period of 5 . As a result, the fundamental frequency $F$ of a tone produced at a sample rate $s$ with a lattice of size $n$ is given by $F=s /(5 n)$. The fifth upper partial (frequency $s / n$ ) is particularly strong and can be heard distinctly above the fundamental. (Refer to Sound Examples 23 and 24.)

\section{Sine Wave Initialization}

Interesting results can be obtained by setting the initial configuration to

$$
x_{i}(0)=\frac{1}{2}(\sin (2 \pi m i / n)+1), \quad i=0, \ldots, n-1,
$$

where $m$ is a positive integer parameter. Note that with identity map $f$ and $\epsilon=0$, this initial configuration would result in a sine wave with frequency $\mathrm{sm} / \mathrm{n}$. In contrast, with a random initial configuration, which approximates a range of frequencies at equal strength, this initialization uses a single frequency.

Usig the circle map $f$ (of the form in Equation 5), and a sufficiently large lattice size (n greater than about 10,000 ), this setting creates a rhythmically pulsing tone. With a given sample rate $s$, and $m$ and $n$, the tone has frequency $f=s m / n$. The rhythm is determined by the dynamic parameters $\omega, K$, and $\epsilon$. As a rule of thumb, if $\omega=p / q$ is a rational number represented by a reduced fraction, then the rhythm can be interpreted as having $q$ beats per measure, with tempo $s / n$. Because of rounding errors, the pulsing gradually devolves into noise. This "descent into chaos" is particularly striking when $m=100$ and $f$ is the circle map with parameters given by Equation 6. Sound Examples 25 and 26 use the parameters provided in Table 1.

\section{Zero Initialization}

If the system is synchronized initially, as when $x_{i}(0)=0$ for all $i$, then it remains synchronized forever. Therefore, the system reduces to the 
Table 1. Parameter Values for Sine Wave Initializations

\begin{tabular}{lrrrrr}
\hline & $n$ & $m$ & $\omega$ & $K$ & $\epsilon$ \\
\hline Example 1 & 10,000 & 10 & $5 / 9$ & 1 & 0.3 \\
Example 2 & 10,000 & 10 & $3 / 7$ & 1 & 0.3 \\
Example 3 & 200 & 1 & $5 / 9$ & 1 & 0.3 \\
Example 4 & 10,000 & 100 & $5 / 9$ & 1 & 0.3 \\
\hline
\end{tabular}

iteration of the map $f$, and the coupling strength $\epsilon$ has no influence. This allows us to provide a simple example of a sound generated by CML that has spatial simplicity but still produces noise. This is illustrated by the circle map with parameters $\omega=5 / 9$ and $K=2.5$. The generated sounds make it clear that the temporal chaos "drowns out" the spatial order. This is especially pronounced with smaller lattice sizes, as shown by Sound Examples 29 and 30.

\section{Conclusions and Future Directions}

We have explored the use of complex nonlinear dynamics as a sound generator. Specifically, we used one-dimensional coupled map lattices, which combine the iteration of a nonlinear map with linear local averaging. We presented a method of synthesizing sound from the resulting dynamics by using variables at all spatial locations in the lattice order (and also in temporal order). In a typical case, rapid convergence to an equilibrium produces a steady tone. The spatial organization of the steady state determines a characteristic timbre of this tone, with subtle variations due to different initial conditions. Moreover, the size (i.e., the length) of the network determines the pitch. From this perspective, such a mathematical object is an abstract form of a musical instrument.

We have limited our exploration to several case studies with a linear, logistic, or circle map.

The linear case emulates the sound of a plucked string, with detectable alteration when the coupling strengths are chosen at random. The behavior induced by our first nonlinear function, the logistic map, is as expected: As the nonlinearity increases, the resulting sound interpolates between a pitched tone and noise.

By far, the most versatile is the circle map, for which we have provided four particularly interesting choices for its three parameters. We have given two examples with embedded particle systems, one with annihilating diffusions with two competing equilibria and another with repelling particles that produces a steadily changing timbre. Another circle map case exhibits nearly periodic behavior with a resulting combination of frequencies. Our leading circle map example converges to its equilibrium very slowly, providing an opportunity to hear a sonic representation of a metastable strange attractor, which turns out to resemble drone music. The same parameter choice also produces, when initialized by a sine wave, a melody that gradually devolves into noise.

Our results open several avenues for further exploration. Clearly, one could look for new features using different functions $f$ from those that were investigated here. Perhaps a greater priority is to fully understand the potential of the circle map with its three parameters $\epsilon, \omega$, and $K$. Indeed, our most interesting examples were obtained by little more than educated guessing. An "atlas" of parameter space with a catalog of behaviors would be expedient if one wanted to contemplate possible applications to, say, sound effects in computer games. Particularly the role of the coupling strength $\epsilon$ is mysterious and counterintuitive, and it deserves additional investigation. Furthermore, we only touched on the effects of spatial heterogeneity: Either $\epsilon$ or a parameter in the function $f$ could vary from location to location (e.g., by a random choice). Temporal heterogeneity is another possibility: The update rule may change abruptly or gradually-for example, to create a tone decay. Finally, adding random noise to the update rule may test the stability of some of the observed behaviors.

\section{Acknowledgments}

We thank the reviewers and the editor for their constructive comments, which significantly improved the presentation. Both authors were partially 
supported by NSF grant DMS-1513340. Janko Gravner also received partial support through Simons Foundation Award no. 281,309 and the Republic of Slovenia's Ministry of Science program P1-285.

\section{References}

Acebrón, J. A., et al. 2005. "The Kuramoto Model: A Simple Paradigm for Synchronization Phenomena." Reviews of Modern Physics 77(1):137-185.

Battey, B. 2004. "Musical Pattern Generation with Variable-Coupled Iterated Map Networks." Organised Sound 9(2):137-150.

Burraston, D., and E. Edmonds. 2005. "Cellular Automata in Generative Electronic Music and Sonic Art: A Historical and Technical Review." Digital Creativity 16(3):165-185.

Chareyron, J. 1990. "Digital Synthesis of Self-Modifying Waveforms by Means of Linear Automata." Computer Music Journal 14(4):25-41.

Dzjaparidze, M. 2015. "Exploring the Creative Potential of Physically Inspired Sound Synthesis." PhD dissertation, Queen's University Belfast.

Hizanidis, J., et al. 2016. "Chimera-like States in Modular Neural Networks." Scientific Reports 6:Art. 19845. Available online at www.nature.com/articles /srep19845. Accessed February 2018.

Kaneko, K. 1993. "The Coupled Map Lattice." In K. Kaneko, ed. Theory and Applications of Coupled Map Lattices. New York: Wiley, pp. 1-49.

Karplus, K., and A. Strong. 1983. "Digital Synthesis of Plucked String and Drum Timbres." Computer Music Journal 7(3):43-55.

Miranda, E. R. 1995. "Granular Synthesis of Sounds by Means of a Cellular Automaton." Leonardo 28(4):297300.

Miranda, E. R. 2007. "Cellular Automata Music: From Sound Synthesis to Musical Form." In E. R. Miranda and J. A. Biles, eds. Evolutionary Computer Music. Berlin: Springer, pp. 170-193.
Serquera, J., and E. R. Miranda. 2011. "Cellular Automata Dynamic Control for Sound Design with Histogram Mapping Synthesis and the Multitype Voter Model." In Proceedings of the International Computer Music Conference, pp. 139-142.

Serquera, J., and E. R. Miranda. 2014. "Histogram Mapping Synthesis: A Cellular Automata-Based Technique for Flexible Sound Design." Computer Music Journal 38(4):38-52.

Stefanakis, N., M. Abel, and A. Bergner. 2015. "Sound Synthesis Based on Ordinary Differential Equations." Computer Music Journal 39(3):25-43.

Stein, D. L., and C. M. Newman. 2013. Spin Glasses and Complexity. Princeton, New Jersey: Princeton University Press.

Stoica, P., and R. Moses. 2005. Spectral Analysis of Signals. Upper Saddle River, New Jersey: Prentice Hall.

\section{Appendix: Parameters in Examples of Melodies}

We provide three examples of melodies:

Sound Example A1: To play "In the Hall of the Mountain King" by Edvard Grieg, from his incidental music to Peer Gynt, we use the circle map with $\omega=0.5$ and $K=0.1$ throughout the excerpt with three different values of $\epsilon$, starting with $\epsilon=0.6$, switching a third of the way through to $\epsilon=0.65$, and in the final third to $\epsilon=0.7$.

Sound Example A2: The opening to Ludwig van Beethoven's Fifth Symphony is played using the logistic map with different parameter values for each note. So there are too many values to list.

Sound Example A3: Finally, the sequence of notes for a generated melody was produced by a computer algorithm unrelated to the topic of this article. It is played using the circle map with parameters $\omega=0.13, K=0.9$, and $\epsilon=0.3$. 